\title{
Effects of Moderate Exercise Training on Spatial Behavior Among Old Mice - Preliminary Results
}

\author{
E. Carmeli ${ }^{*}, 1$, B. Imam² and E. Kodesh ${ }^{3}$ \\ ${ }^{I}$ Department of Physical Therapy, Faculty of Social Welfare and Health Sciences University of Haifa, Israel \\ ${ }^{2}$ Rehabilitation Research Lab. GF Strong Rehab Centre4255 Laurel Street. Vancouver, B.C., V5Z 2G9, Canada \\ ${ }^{3}$ Department of Physical Therapy, Faculty of Social Welfare and Health Sciences, University of Haifa, Israel
}

\begin{abstract}
Background: Moderate exercise training has innumerable benefits on physical function, cognition and mental health. However, the effects of exercise training on anxiety reduction that results in increased physical activity is much less appreciated. The objective of this study was to examine the effects of moderate exercise training on anxiety-related behaviors that result in increased physical activity.

Methods: Old (17 months) female mice $(\mathrm{n}=6)$, C57B6 were allocated to either a sedentary or a running group that underwent 12 weeks of treadmill running (20 minutes/day, 6 days/week). Anxiety-related behavior was assessed using an Open Field Test.

Results: Moderate exercise training resulted in increased locomotion in the exercised group. These mice entered the 'inner zone' of the open field more frequently; b) exhibited higher movement velocity within the arena; c) traveled a longer distance; and d) spent less time at the corners of the open field.

Conclusion: Our results indicate the beneficial effects of moderate exercise training on reducing anxiety-related behavior and triggering spatial behaviors in an Open Field Test among aged mice.
\end{abstract}

Keywords: Aging, anxiety, mice, running, open field test, spatial behavior.

\section{INTRODUCTION}

Getting older seems to naturally involve a gradual decrease in physical activity. Age-related decline in physical activity leads to both physical and cognitive decline, which in turn can result in functional disability and significantly reduced quality of life [1]. The American College of Sports Medicine (ACSM) has published exercise recommendations in July 2009 for older adults [2]. As a result, awareness of the benefits of physical activity and exercise among the general population and among those with chronic diseases and health limitations due to psychosomatic disorders has gradually increased [3].

Sedentary older adults are at a higher risk for developing chronic illnesses that include metabolic diseases (i.e., diabetes, hypothyroidism, and metabolic syndrome), degenerative joint disease, primary sarcopenia, neurological diseases (i.e., Parkinson's disease, stroke, and Alzheimer's disease), cardiovascular-pulmonary diseases (heart failure, chronic obstructive pulmonary disease, and atherosclerosis) [4-5], cognitive decline and stress and anxiety. Anxiety is a complex psychiatric disorder characterized by affective,

*Address correspondence to this author at the Department of Physical Therapy, Faculty of Social Welfare and Health Sciences University of Haifa, Israel; Tel: 972 507393454; Fax: 972 48288140;

E-mail: ecarmeli@univ.haifa.ac.il cognitive, and physiological impairments that lead to maladaptive behavior. The high prevalence of this disabling condition in older adults as well as the limitations in the existing medications and interventions highlight the need for developing effective treatment plans for this condition. Animal models allow investigation of key biological factors of this disorder. Symptoms of anxiety and stress in mice can be found in the expressions of emotional distress such as irritability and apprehension [6]. Mice display similar aging process to humans (i.e. reduced skeletal muscle mass, strength and diminished regenerative capacity, and sarcopenia) [7]. Studies on sedentary lifestyle in mice have significantly contributed to understanding the major risk factors for human's age-related diseases such as obesity, diabetes, hypertension as well as age-related decline in cognitive abilities [8].

Previous studies suggest that the most effective intervention that ameliorates the deleterious effects of aging and sarcopenia is exercise. Moreover, both in human [9] and rodents [10] regular physical activity contributes to longevity, improves self-confidence and positive emotions, and reduces depression and anxiety. Physical activity reduces the risk of depression and anxiety, improves emotional feeling and enhances self-control [11]. The benefits of physical activity on anxiety, stress, and selfcontrol were estimated commonly by means of self-report questionnaires [12]. Although self-report questionnaires are one of the most efficient methods of collecting these data, 
more objective and reliable methods are required to assess the effect of exercise on anxiety. But given that self-report questionnaire cannot be used in mice, an open-field test was used for anxiety-like behavior assessment. Therefore we measured the effect of moderate exercise training on spatial motor behavior and organization of behavior in time and space as an indication of anxiety or self-confidence level in old mice [13]. Rodents that lack a sense of security as a result of anxiety prefer markedly prolonged stays at the edges or corners of the open field arena [14] while staying in or moving to the center of the arena indicates confidence and less anxiety [15].

\section{MATERIALS AND METHODS}

\section{Subjects}

The study was conducted in Zoological Garden at Tel Aviv University. Six 17-month old female mice (equivalent to $\sim 80$ years old of human being), strain C57BL/6N black, were taken from the animal house at Ichilov Medical Center. Body weight ranged from 18 to $22 \mathrm{~g}$ (standard deviation \pm 1.5). In this strain of mice, females did not show cannibalistic behavior and were not aggressive. They were friendly and therefore 3 animals put in one cage. The mice were randomly allocated using a random number generator into two groups: an exercise group $(\mathrm{n}=3)$ and a sedentary group $(\mathrm{n}=3)$.

\section{Evaluation}

The open-field test (OFT) is commonly used in basic research with rats and mice [16]. This test evaluates the level of emotional stress and anxiety [17]. The test was performed in a square pad (arena) 1.5 x1.5 meters with a radius of $\sim$ 0.75 meter. This surface has smooth walls and is tall enough (wall height is $20 \mathrm{~cm}$ ) so that rodents will not be able to climb out or escape. It is an arena without food or water. The arena located in a quiet room, behind a transparent wall and is monitored by a video camera ( 20 photos per second) connected to a computer program (AccuScan ${ }^{\mathrm{TM}}$, Digiscan photo beam system) that graphically and numerically documents the spontaneous movements of the animal in the peripheral and internal parts (boundaries of outer zone has 15 $\mathrm{cm}$ margin, and inner zones) of the field. The camera system has 16 beams that cover a large surface. Once the animal crossed the scan lines, information is instantaneously transmitted to the computer software, which recorded the animal's location and calculated the speed of moving to another line. All tests were conducted between 9:00 to 10:00 am in partial darkness (dim lighting) and lasted 20 minutes. The test recorded the time (in seconds) that the animal spend in the arena (inner /outer zones) along the walls of the surface and the edges (field walls and corners), as well as the direction, distance $(\mathrm{cm})$ and speed of the movement (distance/time).

Data was collected by a blinded evaluator. All the mice underwent the OFT twice: one day before the exercise program began and one day after the exercise program.

The study hypothesis was that the mice in the exercise group will be less anxious compared to the control mice and as a result will move more and spend less time at the corners of the field.

\section{Intervention}

After three days of familiarization and habituation with the treadmill, resting in a building frame with three separate compartments/cells, one mouse was placed in each compartment/cell. The three separated compartments/cells frame was placed on the treadmill thus allowing three mice to run at the same time. During the three days of acclimation, all mice (including the control mice) were exposed to jogging on the treadmill, adapting to the treadmill noise, human touch and manipulation of slow running (about 7-8 $\mathrm{cm}$ per second for a total of 3, 4 and 5 minutes). The running intervention lasted three months, the activity time took place six days a week at between 9:00 to 10:00 am, 20 minutes each day at the speeds of up to 10 meters per minute, which is equivalent to up $60 \%-70 \% \mathrm{VO}_{2 \mathrm{Max}}$, as formulated by Chappell et al. [18-19].

However, the controls for this type of behavioral experiment were also treated somewhat exactly as the experimental ones with the exception of running. It means that they were taken from the cage every day, kept on a nonmoving treadmill for the same period of time and then put back in the cage. Therefore, all animals were familiarized with touching, people, etc. the same period of time.

\section{Statistical Analysis}

SPSS version 17 was used for statistical analysis. All the mice were evaluated before and after the intervention. Mann Whitney test was used to measure the differences of post and pre intervention (calculated post - pre value for each outcome measure) between groups. The level of statistical significance was set at $\mathrm{p}<0.05$.

\section{ETHICS}

The experiment reported in this manuscript was performed in accordance with the ethical standards of the Helsinki Declaration. The Governmental Ethics Committee No. for this study is $18-615,2012$.

\section{RESULTS}

Figs. $(1,2)$ indicate that the mice in the running group were significantly more motor active than in the mice in the sedentary group. Fig. (1) shows the differences in distance traveled by the mice in two groups before and after the exercise intervention. The running mice traveled a significantly longer distance $(\mathrm{cm})$ than the sedentary group at the end of intervention (median 16.336 vs $11.103 \mathrm{~cm}$, $\mathrm{p}<0.05$ ). Fig. (2) shows that the running group had a significantly higher crawling speed $(\mathrm{cm} / \mathrm{sec})$ than the control group at the end of the exercise training (median 10.28 $\mathrm{cm} / \mathrm{sec}$ vs $9.25 \mathrm{~cm} / \mathrm{sec}, \mathrm{p}<0.05$ ). Fig. (3) shows the differences in pre and post time the mice spent at the corners of the arena. After 3 months, both groups spend more time at the corners compared to the start point however. The difference between pre and post intervention in the time at the corners spent by sedentary mice was longer (Sed: pre $70 \pm 15 \mathrm{sec}$, post $92 \pm 15 \mathrm{sec}$; Exe: pre $48 \pm 12 \mathrm{sec}$, post $60 \pm 17$ $\mathrm{sec})$. 


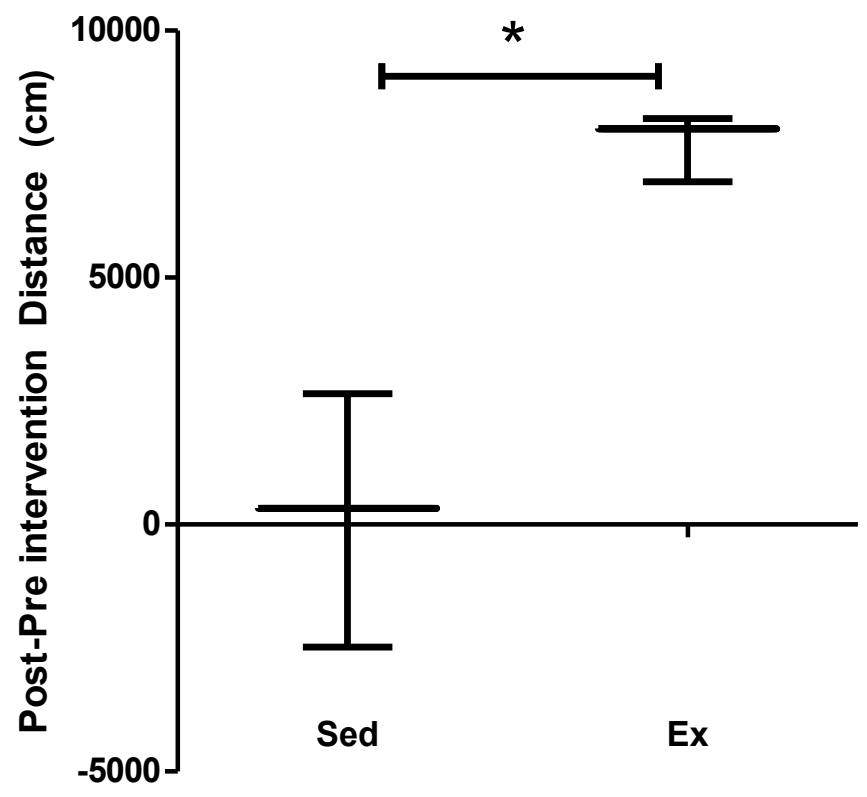

Mann-Whitney test, as a non parametric test, used for paired data.

Fig. (1). Distance $(\mathrm{cm})$ traveled in the arena. Distance difference [Median (range/min-max)] before and after intervention in both groups control (Sed) and exercise (Ex). * Statistically significant between the control and exercise groups $(\mathrm{p}<0.05)$.

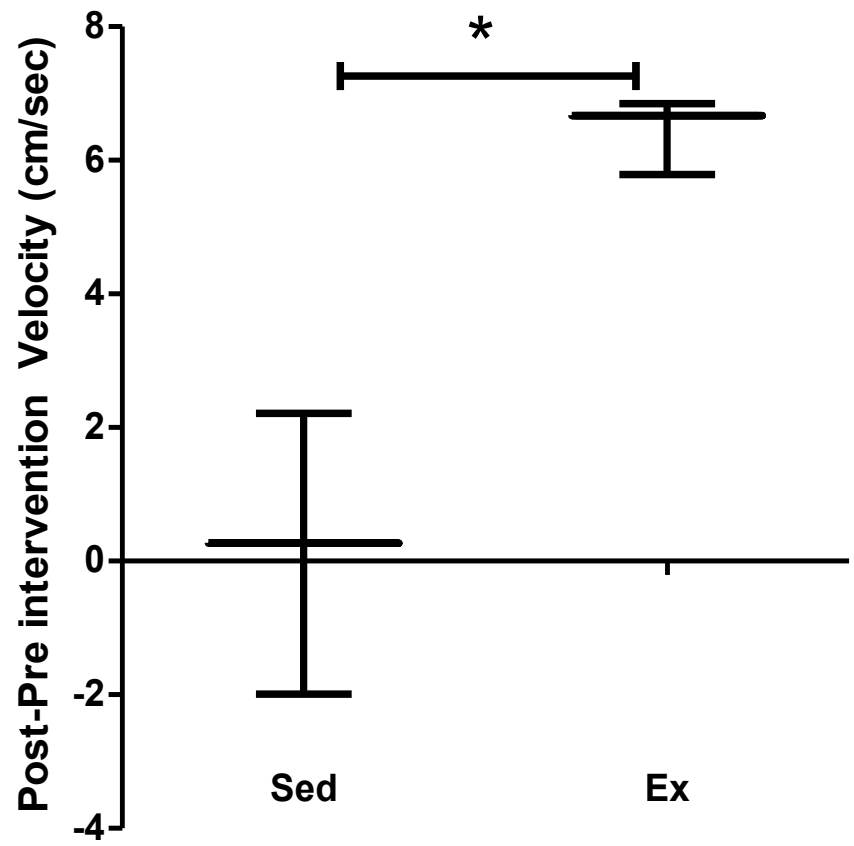

Mann-Whitney test, as a non parametric test, used for paired data.

Fig. (2). Velocity $(\mathrm{cm} / \mathrm{sec})$ Median (range/min-max) in the arena. Velocity difference [Median (range/min-max)] before and after intervention in both groups control (Sed) and exercise (Ex). *Statistically significant between the control and exercise groups, $(\mathrm{p}<0.05)$.

\section{DISCUSSION}

Our results support the study hypothesis that mice exposed to the exercise program would have lower anxiety and therefore exhibit more spatial behavior and movements. We interpret that the increase in movement is a result of the link between a decrease in anxiety level and an increase in spatial behavior following the exercise training [20]. Moreover, the sedentary group spends more time at the arena corners after 3 months of intervention. The preliminary results indicate that those who were exposed to the exercise program perhaps attenuated the effect of aging (3 months period) as expressed by the smaller differences in pre and post time spent at the arena corners.

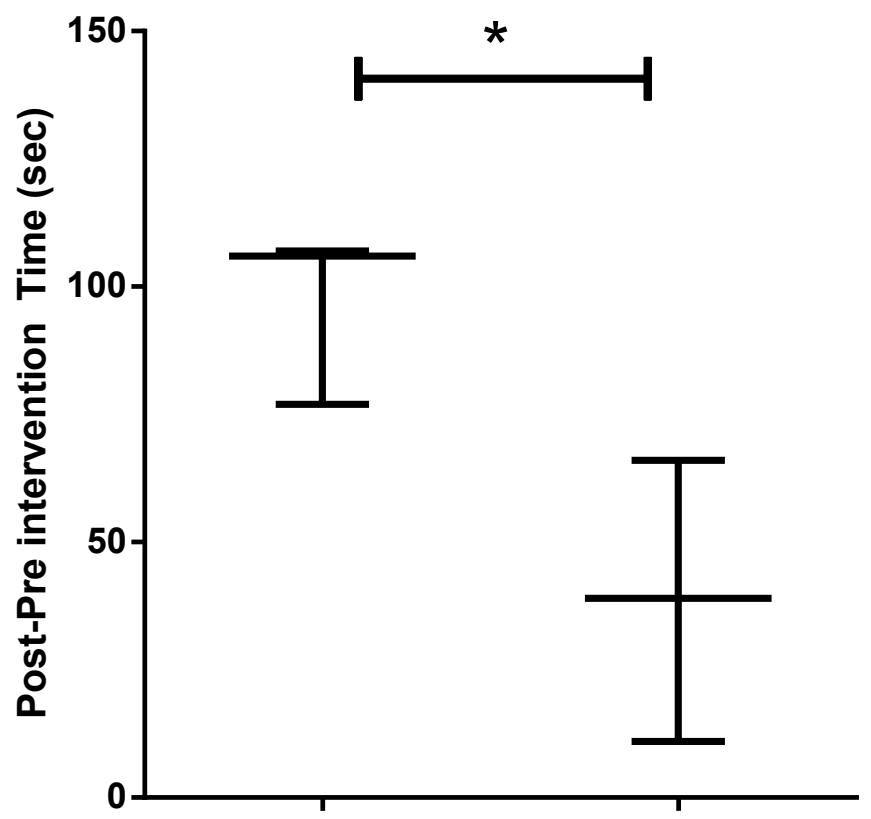

Sed Ex

Mann-Whitney test, as a non parametric test, used for paired data.

Fig. (3). Time (sec) Median (min-max) mice spent at the corners of the arena. Differences of time (sec) [Median (min-max)] before and after intervention, mice spent at the corners of the arena intervention in both groups control (Sed) and exercise (Ex). * Statistically significant between the control and exercise groups, $(\mathrm{p}<0.05)$

Using mice as models has several advantages: it involves a normal, competent, out bred strain, does not involve any treatment, surgery or injection, and allows investigation of the naturalistic development of anxiety while aging.

Anxiety as a function of insecurity or lack of self-control has been evaluated using various laboratory measures. However, the current study shows that spatial behavior after a running program may be a reflection of a decrease in stress or an increase in self-control and confidence. The effect of physical activity on emotional state has been mainly measured using laboratory parameters such as chemical changes in saliva, cortisol [21], blood levels of serotonin and norepinephrine, using physiological parameters of blood pressure, heart rate, cholesterol, oxidative stress and free radicals [22], or using behavioral factors such as sleeping 
and eating [23-24]. However, increase in movement in aged rodents following an exercise program as an indication of decreased anxiety, stress and self-confidence has not documented. Earlier publications indicated that young rodents developed "motor hyperactivity" following a short run, which caused a decrease in the level of stress hormones such as corticosteroids in blood plasma [25], activating "antidepressant" mechanisms, increased levels of serotonin and norepinephrine and creating brain-derived neurotrophic factors [26]. Increase in the amount and speed of locomotion in the OFT and the number of times the running mice entered the center of the arena indicate a sense of security and "sensory-motor inner pressure" [27] to scan and check the open space for different needs (such as food). The increased control and confidence in the running mice resulting from prolonged running can be explained by neurological as well as chemical and neuro-physiological mechanisms. One possible mechanism to explain our results might have to do with an adaptive response in the brain tissue that has led to the expression of brain plasticity. These reactions can trigger growth factors and create new synapses and neuronal connections through neurogenesis, synpathogenesis, angiogenesis and overexpression of certain neuronal chemicals related to anxiety-like behavior [28]. The current findings have empirical and practical implications. First, the results strengthen the argument that exercise training raises the level of locomotion as a result of a decrease in anxiety and stress. The study was conducted on laboratory mice that were fairly old (equivalent to 80-years of age in human beings). Due to the demanding structure of the running program (six days a week, 20 minutes each time for 3 months), two questions should be asked: a) what will be the effect of a shorter intervention or the effect of such running program in younger mice on reducing anxiety level as indicated by locomotion, and b) according to the theory of 'Conservation of Resources' [29], will the increase in locomotion be retained after a physical training program is ended.

The study had a number of limitations. First, because this study just presented preliminary results, it is obvious that the sample size was small which only included female gender, and therefore limits the conclusions. In addition, mice have genetic, biological and behavior characteristics that closely resemble those of humans, and therefore stress symptoms of human conditions can be replicated in rodents [30]. But at the same time, generalizability and validity of our study results to human populations should be taken in great caution especially with regards to emotional aspects; nonetheless many scholars have applied the results of anxiety tests in rodents to human behavior. Further empirical and methodological research is needed to explore the validity and generalizability of the adjusted indirect comparison for evaluating same and different interventions. Future studies, with greater sample of animals, should consider using running wheel to promote exercise, instead of treadmill because it might be a less stressful protocol, and for anxietylike behavior assessment to use 'elevated plus maze' which unfortunately were not available in our lab.

\section{CONCLUSION}

Our results indicate the beneficial effects of moderate exercise training on reducing anxiety-related behavior and triggering spatial behaviors in an OFT among aged mice.

\section{CONFLICT OF INTEREST}

The authors confirm that this article content has no conflict of interest.

\section{ACKNOWLEDGEMENTS}

Declared none.

\section{REFERENCES}

[1] Seekins T, Shunkamolah W, Bertsche M, et al. A systematic scoping review of measures of participation in disability and rehabilitation research: a preliminary report of findings. Disabil Health J 2012; 5: 224-32.

[2] Chodzko-Zajko WJ, Proctor DN, Fiatarone Singh MA, et al. American College of Sports Medicine position stand. Exercise and physical activity for older adults. Med Sci Sports Exercises 2009; 41: 1510-30.

[3] Hallal PC, Andersen LB, Bull FC, Guthold R, Haskell W, Ekelund U. Global physical activity levels: surveillance progress, pitfalls, and prospects. Lancet 2012; 380(9838): 247-57.

[4] Lazarus A, Banerjee KK, Kolthur-Seetharam U. Small changes, big effects: chromatin goes aging. Sub cell Biochem 2012; 61:151-76.

[5] Niccoli T, Partridge L. Ageing as a risk factor for disease. Curr Biol 2012; 22: R741-52.

[6] Nollet M, Le Guisquet AM, Belzung C. Models of depression: unpredictable chronic mild stress in mice. Curr Prot Pharmacol 2013; 5; 50-65

[7] Romanick M, Thompson LV, Brown-Borg HM. Murine models of atrophy, cachexia, and sarcopenia in skeletal muscle. Biochim Biophy Acta 2013; 1832: 1410-20.

[8] Lazarov O, Marr RA. Of mice and men: neurogenesis, cognition and Alzheimer's disease. Front Aging Neurosci 2013; 5: 43-46.

[9] Ferreira ML, Sherrington C, Smith K, et al. Physical activity improves strength, balance and endurance in adults aged 40-65 years: a systematic review. J Physiother 2012; 58: 145-56.

[10] Pietrelli A, Lopez-Costa J, Goni R, Brusco A, Basso N. Aerobic exercise prevents age-dependent cognitive decline and reduces anxiety-related behaviors in middle-aged and old rats. Neurosci 2012; 202: 252-66.

[11] Rogers CE, Larkey LK, Keller C. A review of clinical trials of tai chi and qigong in older adults. West J Nurs Res 2009; 31: 245-79.

[12] Rogers ME, Rogers NL, Takeshima N, Islam MM. Methods to assess and improve the physical parameters associated with fall risk in older adults. Prevent Med 2003; 36: 255-64.

[13] Salam JN, Fox JH, Detroy EM, Guignon MH, Wohl DF, Falls WA. Voluntary exercise in C57 mice is anxiolytic across several measures of anxiety. Behav Brain Res 2009; 197: 31-40.

[14] Fuss J, Ben Abdallah NM, Vogt MA, et al. Voluntary exercise induces anxiety-like behavior in adult $\mathrm{C} 57 \mathrm{BL} / 6 \mathrm{~J}$ mice correlating with hippocampal neurogenesis. Hippocampus 2010; 20: 364-76.

[15] Palanza P, Parmigiani S, vom Saal FS. Effects of prenatal exposure to low doses of diethylstilbestrol, o,p'DDT, and methoxychlor on postnatal growth and neurobehavioral development in male and female mice. Hormon Behav 2001; 40: 252-65.

[16] Keers R, Pedroso I, Breen G, et al. Reduced anxiety and depression-like behaviours in the circadian period mutant mouse afterhours. PLoS One 2012; 7(6): e38263.

[17] Mineur YS, Belzung C, Crusio WE. Effects of unpredictable chronic mild stress on anxiety and depression-like behavior in mice. Behav Brain Res 2006; 175: 43-50.

[18] Chappell MA, Garland T Jr, Rezende EL, Gomes FR. Voluntary running in deer mice: speed, distance, energy costs and temperature effects. J Exper Biol 2004; 207: 3839-54.

[19] Knab AM, Bowen RS, Moore-Harrison T, Hamilton AT, Turner MJ, Lightfoot JT. Repeatability of exercise behaviors in mice. Physiol Behav 2009; 98: 433-40. 
[20] DeBoer LB, Powers MB, Utschig AC, Otto MW, Smits JA. Exploring exercise as an avenue for the treatment of anxiety disorders. Expert Rev Neurother 2012; 12: 1011-22.

[21] Stupnicki R, Obminski Z. Glucocorticoid response to exercise as measured by serum and salivary cortisol. Euro J Appl Physiol Occup Physiol 1992; 65: 546-49.

[22] Agarwal D, Dange RB, Vila J, Otamendi AJ, Francis J. Detraining differentially preserved beneficial effects of exercise on hypertension: effects on blood pressure, cardiac function, brain inflammatory cytokines and oxidative stress. PLoS One 2012; 7: e52569.

[23] Sartori SB, Landgraf R, Singewald N. The clinical implications of mouse models of enhanced anxiety. Future Neurol 2011; 6: 531-71.

[24] van Hulzen ZJ, Coenen AM. Paradoxical sleep deprivation and locomotor activity in rats. Physiol Behav 1981; 27: 741-44.

[25] Duman CH, Schlesinger L, Russell DS, Duman RS. Voluntary exercise produces antidepressant and anxiolytic behavioral effects in mice. Brain Res 2008; 1199: 148-58.
[26] Gao S, Cui YL, Yu CQ, Wang QS, Zhang Y. Tetrandrine exerts antidepressant-like effects in animal models: role of brain-derived neurotrophic factor. Behav Brain Res 2013; 238: 79-85.

[27] Canbeyli R. Sensorimotor modulation of mood and depression: an integrative review. Behav Brain Res 2010; 207: 249-64.

[28] Urakawa S, Takamoto K, Hori E, Sakai N, Ono T, Nishijo H Rearing in enriched environment increases parvalbumin-positive small neurons in the amygdala and decreases anxiety-like behavior of male rats. BMC Neurosci 2013; 14:13-16.

[29] Hobfoll SE. Conservation of resources. A new attempt at conceptualizing stress. Am J Psychol 1989; 44: 513-24.

[30] Karanges E, Li KM, Motbey C, Callaghan PD, Katsifis A, McGregor IS Differential behavioral and neurochemical outcomes from chronic paroxetine treatment in adolescent and adult rats: model of adverse antidepressant effects in human adolescents? Int $\mathrm{J}$ Neuropsychopharmacol 2011; 14: 491-504.

Received: July 7, 2014

(C) Carmeli et al.; Licensee Bentham Open.

This is an open access article licensed under the terms of the Creative Commons Attribution Non-Commercial License (http: //creativecommons.org/licenses/by$\mathrm{nc} / 3.0 /$ ) which permits unrestricted, non-commercial use, distribution and reproduction in any medium, provided the work is properly cited. 